\title{
TOXOPLASMOSIS AND ITS OCULAR MANIFESTATIONS
}

\author{
Kr. Koev \\ Department of Urgency Medicine \\ Medical University - Sofia
}

\begin{abstract}
Summary. The present review provides general information about the parasitic infection of toxoplasmosis and describes the ways of its transmission. It outlines the importance of the consequences of toxoplasmosis infection and the methods of its prevention. The review traces the harmful effects of the disease on human and animal organisms, the causes and stages of development of the disease. The review specifically focuses on the ocular manifestations of toxoplasmosis which can cause ocular lesions, inflammation and scarring. Herein are described the ways toxoplasmosis can damage the eyes causing chorioretinitis, nystagmus, microphthalmia, etc. Furthermore, the review addresses the problem of how congenital and acquired toxoplasmosis affects eyes. The ocular symptoms of toxoplasmosis include weakened or blurred vision, eyeballs pain, ocular sensitivity to light, etc. The harmful effects of toxoplasmosis to pregnant women and immunocompromised patients have been delineated. Some of the disease manifestations include jaundice, rash, asphyxia, etc. The review traces the diagnostic work-up and comments on common tests for toxoplasmosis, such as taking of blood serum samples. The review ends with the treatment of the disease and of its ocular manifestations in particular, for example application of intravitreous injection. The prevention of the infection is extremely important for pregnant women, immunocompromised patients and patients with AIDS.
\end{abstract}

Key words: toxoplasmosis, ocular manivestations

\section{INTRODUCTION}

oxoplasmosis is a widespread reaction to parasites which affects animals and mammals and many types of birds. Human is also affected by this parasitic infection which frequently develops unnoticed or with very mild disease manifestations. The importance of such reactions to parasites comes from its tendency to damage severely the fetus in the womb of pregnant women, should 
contamination occurred during the first 3 to 6 months of pregnancy. In such cases the child is born with congenital toxoplasmosis.

The germ is very small in size. It multiplies inside the cells where it forms cysts, which contain hundreds of parasites. Cats are the main source and the permanent reservoir of infection and may infect human and other animals. The sexual development of the parasite performs in the gut of cats. Animals and humans become infected through the excrements of cats. The parasites can get into the human body through infected animal meat. Cat rearing and consumption of raw or medium-well meat increases the risk of contamination [3].

Herbivores are infected through ingestion of parasitic cysts, which easily fall into the feed and the lawns of feline feces. Harm that parasites cause to human organism affects many organs but most frequently the parasite affects the brain and the inner membranes of the eye. Congenital toxoplasmosis manifests even in newborns with brain and cerebral membranes inflammation, skin rash, fever, lethargy, spleen and liver enlargement, increase or decrease in the size of the head, blindness, jaundice, seizures, etc. These manifestations of toxoplasmosis can happen in the first months of the infant's life, but they can also perform in youth in the form of mental retardation, vision disorder, cerebral palsy, seizures, etc. Infected pregnant women give birth to a child with congenital toxoplasmosis the first time only. After that in consequent pregnancies children are born healthy. Toxoplasmosis acquired after birth in most cases develops without any symptoms. In rare cases fever, muscle pain, transient skin rash, swollen lymph nodes, myocarditis may be noticed. Continuation of disease manifestations may take from several days to several weeks and in most cases results in spontaneous recovery. Prevention is of great importance especially for pregnant women who have to avoid communication with cats and other pets [4].

\section{OCULAR MANIFESTATIONS OF TOXOPLASMOSIS}

The Toxoplasma gondii (T. gondii) parasite, which causes toxoplasmosis, can lie dormant (inactive) in the retina for many years. However, once the parasite becomes active again, it can start a new infection [5].

Ocular toxoplasmosis causes ocular lesions, which are wounds in the eyes caused by inflammation and scarring. These can appear in the: 1) retina - the light-sensitive layer of tissue at the back of the eye; or 2) choroid - the layer behind the retina that contains major blood vessels. This damage to the eyes is called retinochoroiditis and it can cause eye problems such as: a partial loss of eyesight in one eye, squint - where one eye looks in a slightly different direction to the other one, clouding of the eye's lens (cataracts), eye shrinking (microphthalmia), loss of cells and tissue from the optic nerve, which connects the eye to the brain, resulting in poor vision (optic atrophy) [6].

The most common finding in congenital toxoplasmosis is the ophthalmologic manifestation retinochoroiditis, which has a predilection for the posterior pole. It is seen in $75-80 \%$ of cases and is bilateral in $85 \%$ of cases. In acquired toxoplasmosis, 
the ocular form of the disease occurs much less frequently. Previously, only 1-3\% of patients with acquired infection were believed to develop ocular toxoplasmosis. Some of the ocular manifestations of acquired toxoplasmosis are chorioretinitis, iridocyclitis, and uveitis [7].

However, serologic studies suggest that ocular toxoplasmosis is more commonly associated with acquired infection than was previously believed. Ocular lesions in congenital toxoplasmosis include bilateral chorioretinitis and atrophy of the optic nerve. Nystagmus, strabismus and microphthalmia can be only transient manifestations in the course of the disease, occurring through different time after birth.

The above listed severe organ and systemic disabilities are peculiar for congenital toxoplasmosis developing immediately after birth with the following manifestations: chorioretinitis, hydrocephalus, and brain calcifications. In congenital ocular toxoplasmosis blood serum, cerebrospinal fluid, biopsy of the placenta, amniotic fluid and umbilical cord blood are tested [8].

\section{WHICH ARE TOXOPLASMOSIS SYMPTOMS?}

Ocular symptoms of toxoplasmosis infection include chorioretinitis (inflammation of the retina and choroid), weakened or blurred vision, pain in the eyeballs (especially when exposed to strong light), ocular sensitivity to light (photophobia), erythema or watering of the eyes. Treatment application depends on toxoplasmosis stage, localization and severity of eye damage. Ocular lesions may reactivate after years and may progressively lead to blindness [9].

However, toxoplasmosis often manifests without symptoms. In congenital toxoplasmosis newborns sometimes develop myocarditis, pneumonia, nephritis, etc. In more severe infection hydrocephalus, chorioretinitis, optic atrophy, strabismus are observed. Untreated children may develop mental retardation, motor disturbances, seizures, etc. When women suffered toxoplasmosis prior to pregnancy the fetus is not endangered from congenital toxoplasmosis due to the presence of antibodies in the body [9].

In acquired toxoplasmosis, depending on the pathogenesis of the strain and the immune status of organism, the disease can go from asymptomatic to acute and severe. There are three common types of acquired toxoplasmosis - lymphatic, ocular and cerebral. The lymphatic form is characterized with swollen neck, axillary and inguinal lymph nodes. They can reach the size of a walnut and are painless and easily treated. Sometimes there is fever, and the clinical course is similar to that of infectious mononucleosis or Hodgkin's disease. Ocular manifestations of acquired toxoplasmosis include uveitis, chorioretinitis, iridocyclitis [10]. Cerebral manifestations of acquired toxoplasmosis include meningoencephalitis. In patients with immune deficiency myocarditis and pneumonia may also develop. 


\section{TOXOPLASMOSIS IN PREGNANT AND IMMUNOCOMPROMISED PATIENTS}

Some toxoplasmosis symptoms of a general nature are: not very high temperature, long lasting icterus, cyanosis, asphyxia, rash. In rare cases ascites can develop.

Among symptoms of internal organs could be mentioned pneumonia, myocarditis, nephritis, hepatosplenomegaly, enteritis, as well as ocular and central nervous system symptoms.

Central nervous system disorders are severe and can occur after child birth or in a later stage of life [11].

Meningoencephalitis syndrome includes impaired general condition, swallowing disorders, vomiting, high fever, increased muscle tone, ataxia, tremor, paralysis. Seizure syndrome is one of the first manifestations of the disease and it is possible to persist over time. Becoming status epileptucis, it may be the immediate cause of a newborn's death. Another typical localization of the parasite is the choroid plexus which penetrates the cerebral ventricles and causes ependymitis. If the process is unilateral and affects only one hemisphere, patients can develop unilateral hydrocephalus. In the first stage of brain atrophy it is possible microcephaly to occur. The peculiar cerebrospinal fluid changes appear as xanthochromia, pleocytosis and increased protein levels. Damages to the central nervous system may cause retardation in psychomotor development, which is noticeable at the end of the first year of the child's life. Sometimes the mental retardation can not be observed until school age.

In infants, for whom toxoplasmosis infection manifests during the first months of their lives, it can develop severely with fever, rash and jaundice.

In all other cases, the toxoplasmosis infection is lethal in the form of parasitic transmission.

Placenta involvement occurs in $20 \%$ to $40 \%$ of cases of primary toxoplasmosis during pregnancy. This results in an infection of the fetus. Most frequently it develops without any symptoms, but in $10 \%$ to $20 \%$ of the infected children a clinical manifestation after birth is observed [12].

In infants with manifestation of toxoplasmosis during the first months of their lives, the disease may be accompanied by harsh fever, lesion rash, lymphadenopathy, hepatosplenomegaly and jaundice.

It was reported that the reactivation of parasites from tissue cysts located in the retina can cause inflammatory reaction many years after the primary infection, which is characterized with recurrent chorioretinitis, usually occurring after the second decade of life [13].

\section{DIAGNOSIS}

It is important for the diagnosis that we distinguish acute toxoplasmosis from widespread infection in order to carry out adequate etiologic therapy and prophylactic regimen. The clinical diversity of such reactions to parasites requires integrated 
assessment, which should involve epidemiological, clinical, parasitological and serological investigation, all of which representing an important criterion for the exact diagnosis [14].

\section{TREATMENT}

Antibiotics and steroids are often used to treat the lesions. The scarring caused by toxoplasmosis will not clear up, but treatment may prevent it from getting worse.

If the infection keeps returning, antibiotics may be prescribed on a long-term basis. While this may help prevent the infection from reoccurring, the long-term side effects of these medications are not yet known [15].

Chronic carriers of toxoplasmosis can not be cured. Toxoplasmosis of the lymph nodes in immunocompetent patients usually resolves spontaneously without treatment.

Indications for antibiotic treatment include: i) toxoplasmosis with clinical symptoms (such as fever, muscle aches, etc.). ii) primary infection during pregnancy; or iii) immunosuppressed patients and AIDS patients with acute toxoplasmosis.

First choice medication should be pirimetamin (+ folic acid) with sulfadiazine or clindamycin. This treatment is prohibited before the 20th gestational week and in these cases spiramycin (also other macrolides, such as azithromycin) is suitable.

\section{INTRAVITREOUS INJECTION THERAPY FOR OCULAR TOXOPLASMOSIS}

Intravitreous therapy with clindamycin and dexamethasone is safer and more effective compared to the conventional approach, suggests a study in patients with active ocular toxoplasmosis. Ocular toxoplasmosis is one of the most common reasons for uveitis that affects the posterior ocular segment. According to various surveys around $50 \%$ of cases of posterior uveitis is caused by toxoplasmosis infection [3].

Standard therapy with pyrimethamine, sulfadiazine and prednisolone may have significant side effects, the treatment lasts for longer time and the price is increased.

Iranian doctors compared conventional therapy with approach developed by them which included up to 3 injections of $1 \mathrm{mg}$ intravitreous clindamycin and 400 mcg dexamethasone. After 6 weeks the reduction of lesion stretch was similar for the two groups $-57.0 \%$ in the new approach versus $58.4 \%$ in the standard one. In both groups similar improvement of visual acuity was reported. There was found that patients with antitoxoplasmic IgM antibodies responded better to standard therapy while patients with IgM-negative responded better to intravitreous therapy with clindamycin and dexamethasone [4]. During a survey for at least 2 years only 2 eyes of each group had a relapse. There were no reported significant infectionrelated complications such as haemorrhage, endophthalmitis, retina detachment or cataracts. 


\section{PREVENTION}

Prevention of toxoplasmosis is particularly important for pregnant women, patients with seronegative immunocompromised and patients with AIDS. In these cases patients need to avoid eating raw meat and contacts with cats. It is important that pregnant women should be screened and patients with AIDS conduct drug prophylaxis $[1,2,5]$.

The high number of homeless cats contribute to the spread of toxoplasmosis. Therefore, the following recommendations should be adopted as rules:

- Domestic cats should be regularly examined and treated.

- Cats should not be allowed to warehouses with food products.

- Vegetables and fruits should be washed thoroughly before consumption.

- Thermal processing of food (that requires such) should be meticulously carried out.

- Washing of hands and cutlery with soap is mandatory.

\section{REFERENCES}

1. Fo u drinier, F., I. Villena, R. Jaussaud et D. Aubert. Clinical Value of Specific Immunoglobulin E Detection by Enzyme-Linked Immunosorbent Assay in Cases of Acquired and Congenital Toxoplasmosis, 41, 2003, № 4, 1681-1686.

2. Christopher, D. et al. Hunter Immune response and immunopathology during toxoplasmosis Semin Immunopathol. - Semin Immunopathol., 34, 2012, № 6, 793-813.

3. Lou is, M. Toxoplasmosis: a history of clinical observations. - Int. J. Parasitol., 39, 2009, № 8, 895-901.

4. Sterkers, Y., F. Pratlong et S. Albaba. Novel Interpretation of Molecular Diagnosis of Congenital Toxoplasmosis According to Gestational Age at the Time of Maternal Infection, 50, 2012, № 12, 3944-3951.

5. Willers on J r., D. et al. Unusual ocular presentation of acute toxoplasmosis. - Br. J. Ophthalmol., 61, 1977, № 11, 693-698.

6. Ch a rles, J. Kipp, Hemorrhagic Central Chorioretinitis in Non-myopic Eyes: A Clinical Contribution. - Trans. Am. Ophthalmol. Soc., 12, 1909, (Pt 1), 250-266.

7. Turunen, H. J., P. O. Leinikki, K. M. Saari. Demonstration of intraocular synthesis of immunoglobulin $\mathrm{G}$ toxoplasma antibodies for specific diagnosis of toxoplasmicchorioretinitis by enzyme immunoassay. - J. Clin. Microbiol., 17, 1983, № 6, 988-992.

8. Florence Robert-Gangneux, Marie-Laure Dardé. Epidemiology of and Diagnostic Strategies for Toxoplasmosis. - Clin. Microbiol. Rev., 25, 2012, № 3, 583.

9. Villard, O. et al. J Clin Microbiol, Comparison of Enzyme-Linked Immunosorbent Assay, Immunoblotting, and PCR for Diagnosis of Toxoplasmic Chorioretinitis, 41, 2003, № 8, 3537-3541.

10. Connor, G. R. O. The infleunce of hypersensitivity on the pathogenesis of ocular toxoplasmosis. - Trans. Am. Ophthalmol. Soc., 68, 1970, 501-547.

11. Gwendolyn Noble,A. Paul Latkany, Jaroslaw Kusmierczyk, the Toxoplasmosis Study Group, Chorioretinal lesions in mothers of children with congenital toxoplasmosis in the National Collaborative Chicago-based, CongenitalToxoplasmosis Study. - Sci. Med. (Porto Alegre), 20, 2010, № 1, 20-26. 
12. M c L e o d, R. et al. Mem Inst Oswaldo Cruz, Why prevent, diagnose and treat congenital toxoplasmosis? - Mem. Inst. Oswaldo Cruz., 104, 2009, № 2, 320-344.

13. Stillwagg o n, E. et al. PLoS Negl Trop Dis, Maternal Serologic Screening to Prevent Congenital Toxoplasmosis: A Decision-Analytic Economic Model, 5, 2011, № 9, e1333.

14. M c Le od, R. et al. Why prevent, diagnose and treat congenital toxoplasmosis? - Mem. Inst. Oswaldo Cruz., 104, 2009, № 2, 320-344.

15. Young-Hoon Park, Ho-Woo Nam, Clinical Features and Treatment of Ocular Toxoplasmosis. Korean J. Parasitol., 51, 2013, № 4, 393-399.

Corresponding author:

Assoc. Prof. Krasimir Koev, MD

Department of Urgency Medicine

UMHAT "Tsaritsa Yoanna"

8 Byalo More St.

Bg - 1527 Sofia

e-mail: k007@abv.bg 